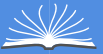

Global Journals Inc.

(n)

का

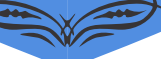

2

\title{
Unconstrained Quadratic Programming Problem with Uncertain Parameters
}

By Sie Long Kek, Fong Peng Lim \& Harley Ooi

Abstract- In this paper, an unconstrained quadratic programming problem with uncertain parameters is discussed. For this purpose, the basic idea of optimizing the unconstrained quadratic programming problem is introduced. The solution method of solving linear equations could be applied to obtain the optimal solution for this kind of problem. Later, the theoretical work on the optimization of the unconstrained quadratic programming problem is presented. By this, the model parameters, which are unknown values, are considered. In this uncertain situation, it is assumed that these parameters are normally distributed; then, the simulation on these uncertain parameters are performed, so the quadratic programming problem without constraints could be solved iteratively by using the gradient-based optimization approach. For illustration, an example of this problem is studied. The computation procedure is expressed, and the result obtained shows the optimal solution in the uncertain environment. In conclusion, the unconstrained quadratic programming problem, which has uncertain parameters, could be solved successfully.

Keywords: quadratic programming, gradient approach, uncertain parameters, risk simulator, the system of linear equations.

\section{GJRE-I Classification: FOR Code: 010399}

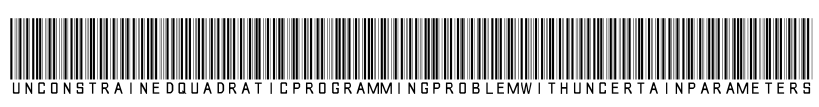

Strictly as per the compliance and regulations of:

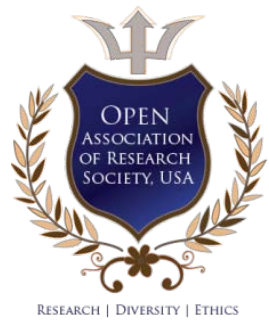

(c) 2021. Sie Long Kek, Fong Peng Lim \& Harley Ooi. This research/review article is distributed under the terms of the AttributionNonCommercial-NoDerivatives 4.0 International (CC BY-NC-ND 4.0). You must give appropriate credit to authors and reference this article if parts of the article are reproduced in any manner. Applicable licensing terms are at https://creativecommons.org/licenses/by-nc-nd/4.0/. 


\title{
Unconstrained Quadratic Programming Problem with Uncertain Parameters
}

\author{
Sie Long Kek ${ }^{\alpha}$, Fong Peng Lim ${ }^{\sigma} \&$ Harley Ooi ${ }^{\rho}$
}

\begin{abstract}
In this paper, an unconstrained quadratic programming problem with uncertain parameters is discussed. For this purpose, the basic idea of optimizing the unconstrained quadratic programming problem is introduced. The solution method of solving linear equations could be applied to obtain the optimal solution for this kind of problem. Later, the theoretical work on the optimization of the unconstrained quadratic programming problem is presented. By this, the model parameters, which are unknown values, are considered. In this uncertain situation, it is assumed that these parameters are normally distributed; then, the simulation on these uncertain parameters are performed, so the quadratic programming problem without constraints could be solved iteratively by using the gradient-based optimization approach. For illustration, an example of this problem is studied. The computation procedure is expressed, and the result obtained shows the optimal solution in the uncertain environment. In conclusion, the unconstrained quadratic programming problem, which has uncertain parameters, could be solved successfully.
\end{abstract}

Keywords: quadratic programming, gradient approach, uncertain parameters, risk simulator, the system of linear equations.

\section{InTRODUCTION}

$\mathrm{n}$ nonlinear optimization, quadratic programming is the most simple optimization problem, and its applications have been widely studied [1], which are ranged from engineering $[2,3,4]$ to business $[5,6,7]$. For allocating resources, the quadratic programming problem, which has an objective function in the quadratic form and subject to a set of constraints, is employed. Consequently, a quantitative decision could be made by referring to the optimal solution obtained from solving the quadratic programming problem, especially with the fuzzy parameters $[8,9,10]$. Also, the computational techniques for solving the quadratic programming problem under the probabilistic environment [11] and the related robust solution [12] are actively studied.

By the use of quadratic programming, this paper aims to discuss the uncertain parameters, which are presented in quadratic programming problem

Author a: Universiti Tun Hussein Onn Malaysia, Pagoh Campus, Muar, Johor, Malaysia.e-mail: slkek@uthm.edu.my

Author o: Universiti Putra Malaysia, Serdang, Seri Kembangan,

Selangor, Malaysia. e-mail: fongpeng@upm.edu.my

Author p: Edustats Solutions, Kuala Lumpur, Malaysia.

e-mail: harley@edustats.com.my without constraints. In doing so, a general unconstrained quadratic programming problem $[13,14$, $15,16]$ is considered, and the analytical solution is discussed. In the uncertain parameters, the analytical solution does not exist unless an assumption of knowing these uncertain parameters is made. Hence, the quadratic programming problem could be handled practically, where the uncertain parameters are assumed to be normally distributed. On this basis, the simulation [17] is performed on the uncertain parameters to give a possible optimal solution to the quadratic programming problem.

The paper is organized as follows. In Section 2, the unconstrained quadratic programming problem is described in general, and the analytical solution is provided. In Section 3, the presence of the uncertain parameters in the quadratic programming problem is taken into consideration. These parameters are assumed from the normal distribution, and the simulation is made to identify these parameters. The solution procedure is then summarized. In Section 4, an illustrative example is further discussed. Finally, a concluding remark is made.

\section{Problem Statement}

Consider a general unconstrained quadratic programming problem $[13,14]$, given by

Minimize $\quad f(\mathbf{x})=\mathbf{x}^{\mathrm{T}} \mathbf{A} \mathbf{x}+\mathbf{b}^{\mathrm{T}} \mathbf{x}+c$

where $\mathbf{X} \in \mathfrak{R}^{n}$ is an $n$-vector of decision variables, $\mathbf{A} \in \mathfrak{R}^{n \times n}$ is a symmetry positive definite matrix, $\mathbf{b} \in \mathfrak{R}^{n}$ is an $n$-vector of coefficients, and $c \in \mathfrak{R}$ is a constant. Under the certainty situation, the values for parameters A, b, and $c$ are known and complete. Thus, to determine the optimal solution of the problem in (1), the first-order necessary condition $[15,16]$,

$$
\nabla f(\mathbf{x})=\mathbf{0}
$$

is derived from giving

$$
\mathbf{A x}+\mathbf{b}=\mathbf{0} .
$$

Note that (3) is a system of linear equations, and its solution can be obtained from 


$$
\mathbf{x}=-\mathbf{A}^{-1} \mathbf{b} .
$$

However, the analytical solution given by (4) would not exist if the parameters $A, b$, and $c$ are unknown and incomplete information. From this point of view, a computational procedure is required to handle these uncertain parameters, so the unconstrained quadratic programming could be solved in practice.

\section{ili. Optimization Modelling Approach}

Now, assume that the uncertain parameters are normally distributed, where mean and covariance are known necessarily $[17,18,19]$. Therefore, a simulation procedure is properly performed in handling these uncertain parameters. It is followed by using any optimization gradient techniques to solve the unconstrained quadratic programming problem for the possible optimal solution.

\section{a) Uncertain Parameters Simulation}

Suppose the values of the elements in the matrix $\mathbf{A}=\left(a_{i j}\right)$ and the vector $\mathbf{b}=\left(b_{i}\right)$, for $i=1,2, \cdots, n$, and $j=1,2, \cdots, n$, are unknown, and these values are defined in the uncertainty set $U$, that is,

$$
U=\left\{a_{i j}, b_{i}\right\}
$$

where

$$
a_{i j} \sim \mathrm{N}\left(m_{a}, \sigma_{a}^{2}\right) \text { and } b_{i} \sim \mathrm{N}\left(m_{b}, \sigma_{b}^{2}\right)
$$

are assumed to be normally distributed with $m_{a}$ is the mean vector and $\sigma_{a}^{2}$ is the covariance matrix for the elements of the matrix $\mathbf{A}=\left(a_{i j}\right)$, and $m_{b}$ is the mean vector and $\sigma_{b}^{2}$ is the covariance matrix for the elements of the vector $\mathbf{b}=\left(b_{i}\right)$

By considering the simulation of the normal distribution, the uncertain parameters are determined from

$$
\begin{gathered}
a_{i j}=m_{a}+z_{a, \alpha} \sigma_{a} \\
b_{i}=m_{b}+z_{b, \alpha} \sigma_{b}
\end{gathered}
$$

where $\alpha$ is the confidence level, ${ }^{z_{a, \alpha}}$ is the $z$-score at the confidence level $\alpha$ for the parameters $a_{i j}$, and $z_{b, \alpha}$ is the z-score at the confidence level $\alpha$ for the parameters $b_{i}$.

\section{b) Calculation Solution Procedure}

After the simulation is carried out to the uncertain parameters, the unconstrained quadratic programming problem is solved by using the optimization gradient approach $[13,15]$, which is given by

$$
\mathbf{x}^{(k+1)}=\mathbf{x}^{(k)}+\beta_{k} \cdot \nabla f^{(k)}
$$

where $\beta_{k}$ represents the step size at the iteration number $k$, calculated from

$$
\beta_{k}=\arg \min f\left(\mathbf{x}^{(k)}+\beta \cdot \nabla f\left(\mathbf{x}^{(k)}\right)\right)
$$

and

$$
\nabla f^{(k)}=\nabla f\left(\mathbf{x}^{(k)}\right)
$$

is the gradient function at $\mathbf{x}^{(k)}$, that is,

$$
\nabla f\left(\mathbf{x}^{(k)}\right)=\mathbf{A} \mathbf{x}^{(k)}+\mathbf{b} \text {. }
$$

From the discussion above, the calculation procedure for solving the unconstrained quadratic programming problem, where uncertain parameters exist, is summarized as follows.

\section{Algorithm 1}

Data A, b, $c, m_{a}, \sigma_{a}, m_{b}$, and $\sigma_{b}$. Set the iteration number $k=0$, the initial value $\mathbf{x}^{(0)}$, the tolerance $\varepsilon$, and the number of simulation trials $n$.

Step 1 Simulate the uncertain parameters $a_{i j}$ and $b_{i}$, respectively, from (7) and (8).

Step 2 Calculate the gradient from (12).

Step 3 Compute the parameter $\beta_{k}$ from (10).

Step 4 Obtain the optimal solution $\mathbf{x}^{(k+1)}$ from (9).

Step 4 Evaluate the value of the objective function $f\left(\mathbf{x}^{(k+1)}\right)$ from (1).

Step 5 Update the simulation trial number. If $\left\|\nabla f^{(k)}\right\|<\varepsilon$, stop the calculation, else, set $k=k+1$, and repeat from Step 1.

Remark

The optimal solution to the unconstrained quadratic programming problem would be different for each simulation cycle. Hence, the simulation values for the decision variable are determined by solving the unconstrained quadratic problem. For this reason, the possible optimal solution shall be preferred in this situation. 


\section{Illustrative Example}

Consider a quadratic programming problem with no constraint $[14,16]$ given by

$$
\begin{aligned}
\text { Minimize } & f\left(x_{1}, x_{2}, x_{3}\right) \\
= & \frac{5}{2} x_{1}^{2}+\frac{7}{2} x_{2}^{2}+3 x_{3}^{2}+x_{1} x_{2}+3 x_{1} x_{3} \\
& +2 x_{2} x_{3}+4 x_{1}+9 x_{2}+8 x_{3}+10
\end{aligned}
$$

This problem is solved by using the risk simulator software $[18,19,20]$. With the complete information of the matrix $A$ and the vector $b$, the optimal solution is $(0.0,-1.0,-1.0)^{\mathrm{T}}$, and the objective function has a minimum value of 1.50 .

For the uncertain situation, two cases are further investigated. In Case 1, only the matrix $A$ is assumed to have an uncertain value of entries, while in Case 2, both matrix $A$ and vector $\mathrm{b}$ are assumed to have uncertain entries. The number of simulation trials is set to 500 , and the number of optimization runs is set to 20 .

Case 1:

Assume that the parameters in matrix $\mathrm{A}$ have mean and standard deviation given by

$$
m_{a}=\left(\begin{array}{lll}
5 & 1 & 3 \\
1 & 7 & 2 \\
3 & 2 & 6
\end{array}\right) \quad \sigma_{a}=\left(\begin{array}{ccc}
0.5 & 0.1 & 0.3 \\
0.1 & 0.7 & 0.2 \\
0.3 & 0.2 & 0.6
\end{array}\right)
$$

Suppose $\mathbf{b}=(4,9,8)^{\mathrm{T}}$ and $c=10$ are known. The simulation results for the decision variables and the objective function are shown in Table 1 and Table 2.

Table 1: Simulation Result for Decision Variables, Case 1

\begin{tabular}{|c|c|c|c|}
\hline $\begin{array}{c}\text { Decision } \\
\text { Variable }\end{array}$ & Initial Value & Final Value & Mean Value \\
\hline$x_{1}$ & -0.00105 & 0.00068 & 0.000277 \\
\hline$x_{2}$ & -1.00107 & -1.01098 & -1.00118 \\
\hline$x_{3}$ & -0.99732 & -0.99795 & -0.99865 \\
\hline
\end{tabular}

Table 2: Simulation Result for Objective Function, Case 1

\begin{tabular}{|c|c|c|c|}
\hline $\begin{array}{c}\text { Objective } \\
\text { Function }\end{array}$ & Initial value & Final Value & $\begin{array}{c}\text { Iteration } \\
\text { Numbers }\end{array}$ \\
\hline$f$ & 1.46040 & 1.46006 & 6 \\
\hline
\end{tabular}

The graphical result for the objective function $f$ is shown in Fig. 1, while the simulation values for decision variables $X_{1}, X_{2}$, and $X_{3}$ are shown in Figs. 2, 3, and 4 , respectively. Notice that the figures' bars represent the respective simulation values, and the lines show the relative cumulative distribution values.

Case 2:

To observe the possible results that might be obtained under the uncertainty, consider the entries of the matrix $\mathbf{A}$ has mean and covariance as given in (14), and assume the entries of the vector $b$ is normally distributed with mean and variance given by

$$
m_{b}=\left(\begin{array}{l}
4 \\
9 \\
8
\end{array}\right) \quad \sigma_{b}=\left(\begin{array}{l}
0.4 \\
0.9 \\
0.8
\end{array}\right)
$$

Table 3 and Table 4 show the simulation results for the decision variables and the objective function, respectively.

Table 3: Simulation Results for Decision Variables, Case 2

\begin{tabular}{|c|c|c|c|}
\hline $\begin{array}{c}\text { Decision } \\
\text { Variable }\end{array}$ & Initial Value & Final Value & $\begin{array}{c}\text { Mean } \\
\text { Value }\end{array}$ \\
\hline$x_{1}$ & -0.01137 & -0.00226 & -0.0019 \\
\hline$x_{2}$ & -1.00806 & -0.99402 & -1.00016 \\
\hline$x_{3}$ & -0.97891 & -0.98902 & -0.99607 \\
\hline
\end{tabular}

Table 4: Simulation Result for Objective Function, Case 2

\begin{tabular}{|c|c|c|c|}
\hline $\begin{array}{c}\text { Objective } \\
\text { Function }\end{array}$ & Initial value & $\begin{array}{c}\text { Final } \\
\text { Value }\end{array}$ & $\begin{array}{c}\text { Iteration } \\
\text { Numbers }\end{array}$ \\
\hline$f$ & 1.63204 & 1.63127 & 9 \\
\hline
\end{tabular}

The graphical result of the objective function and the simulation values for the decision variables $X_{1}$, $X_{2}$, and $X_{3}$ are shown in Figs. 5, 6, 7, and 8, respectively. 


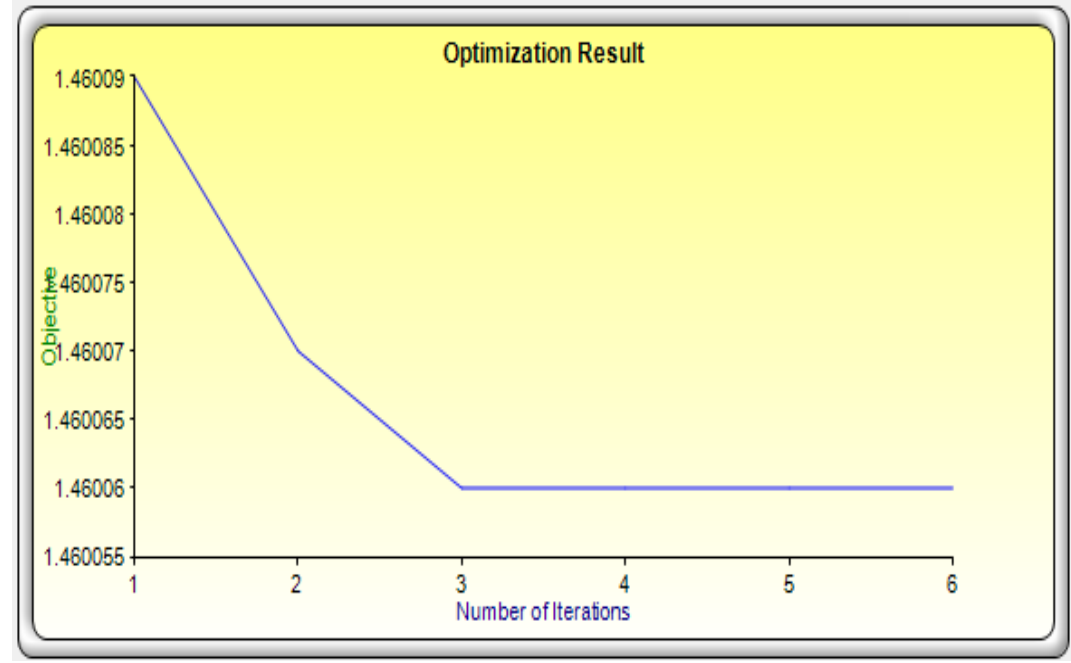

Fig. 1: Objective function, Case 1

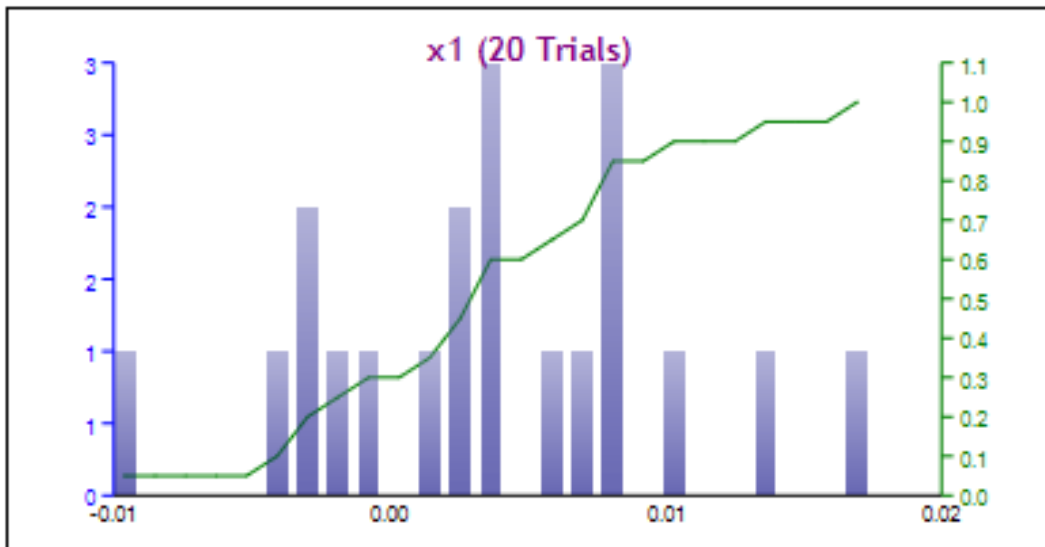

Fig. 2: Simulation values for decision variable $\mathrm{X}_{1}$, Case 1.

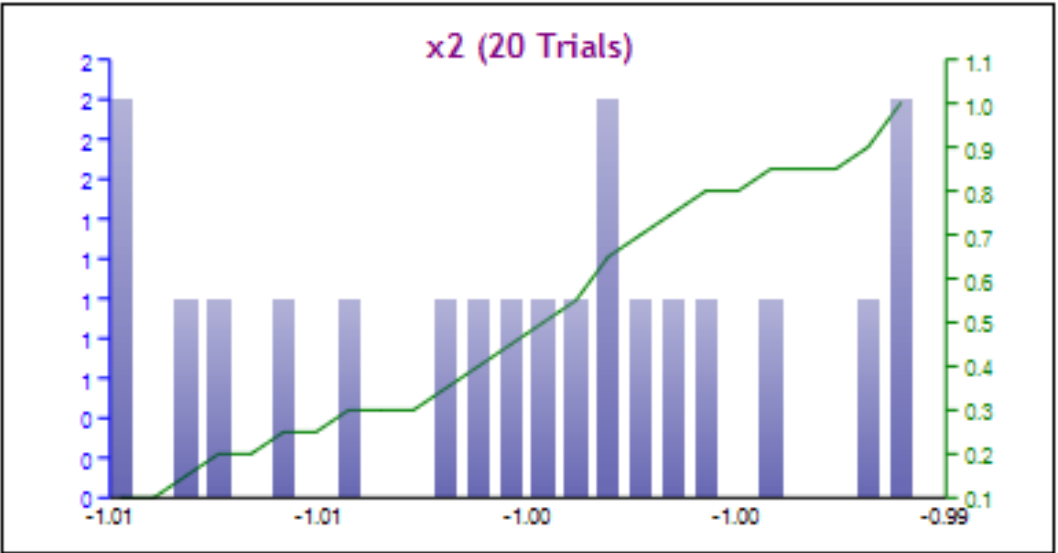

Fig. 3: Simulation values for decision variable $\mathrm{X}_{2}$, Case 1. 


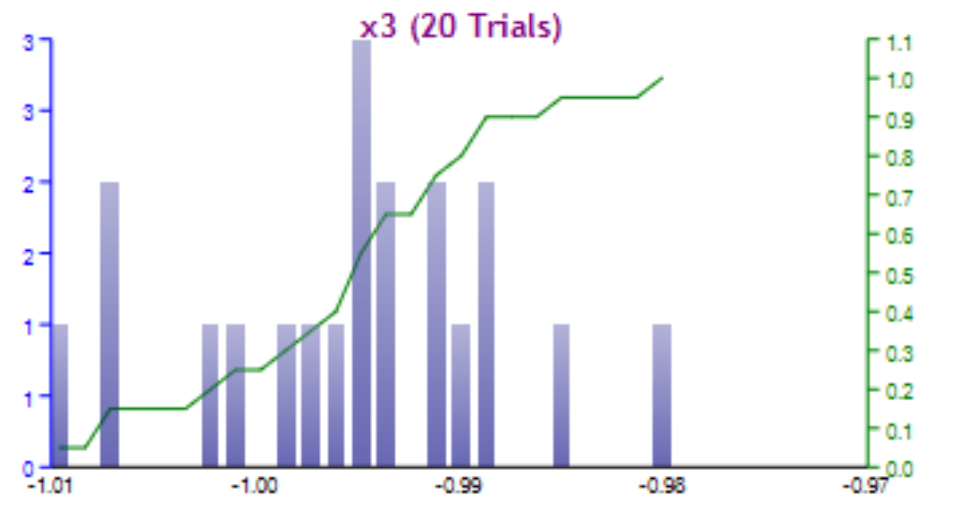

Fig. 4: Simulation values for decision variable $\mathrm{X}_{3}$, Case 1.

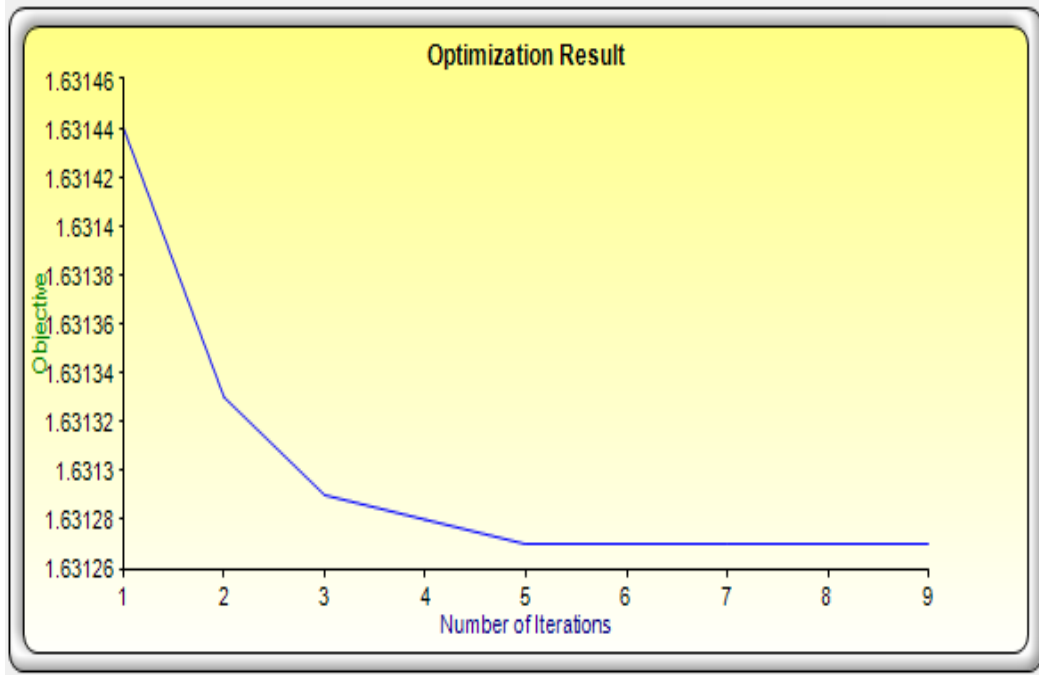

Fig. 5: Objective function, Case 2.

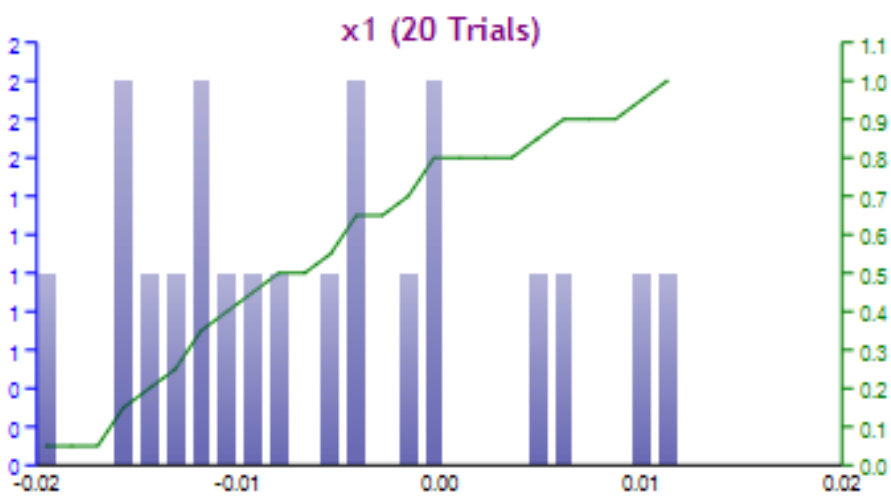

Fig. 6: Simulation values for decision variable $X_{1}$, Case 2. 


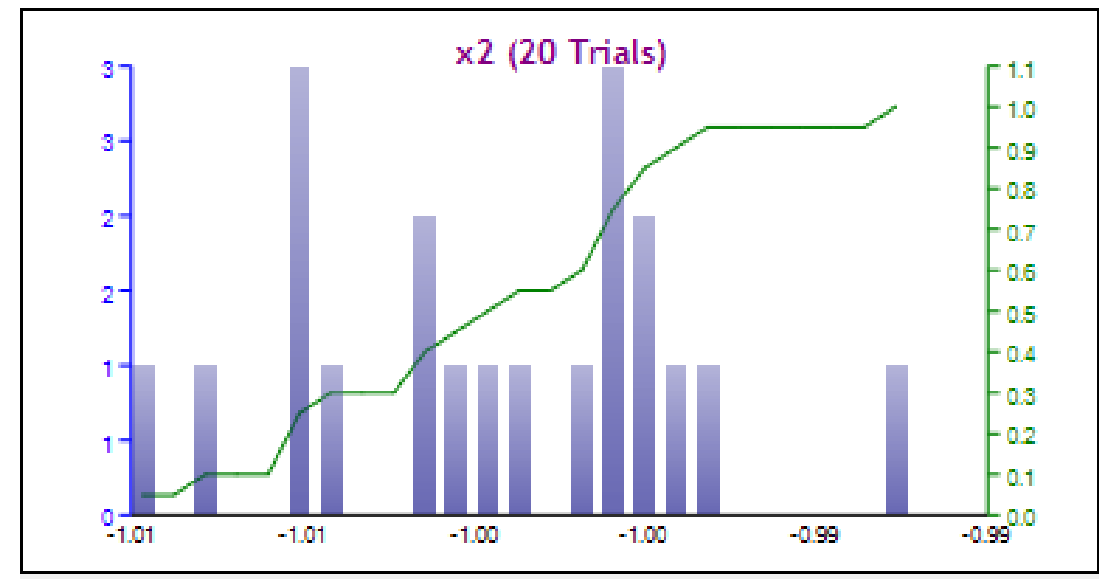

Fig. 7: Simulation values for decision variable $\mathrm{X}_{2}$, Case 2.

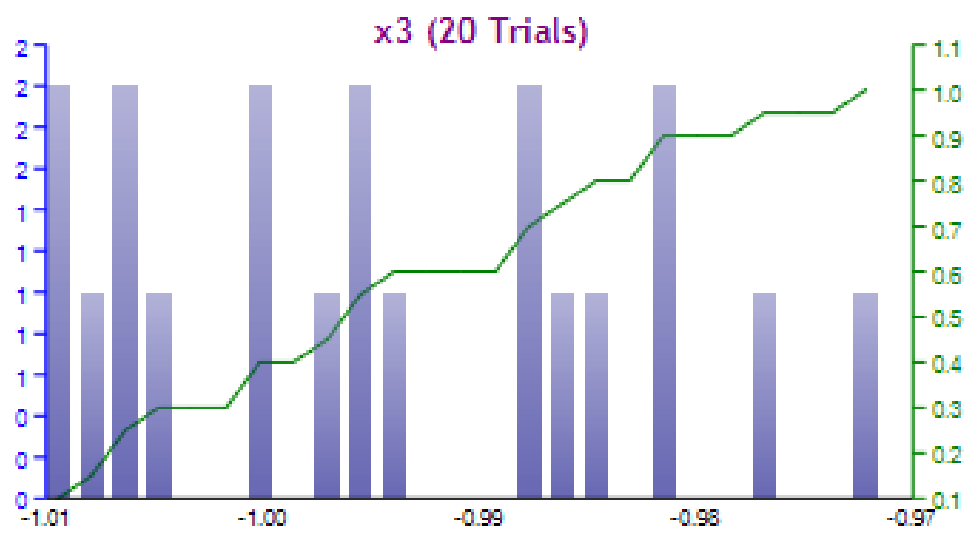

Fig. 8: Simulation values for decision variable $X_{3}$, Case 2.

Therefore, assuming the uncertain parameters are normally distributed and applying the simulation procedure, the unconstrained quadratic programming problem could be solved in advance. The possible optimal solution obtained mainly depends on the simulation performed to the uncertain parameters and the gradient approach used.

\section{Concluding Remarks}

This paper has discussed the unconstrained quadratic programming problem with the presence of uncertain parameters. It is assumed that these parameters are normally distributed, and their values are determined from the simulation process. Accordingly, the unconstrained quadratic programming problem is solved by using the optimization gradient approach. The resulted simulation values of decision values are identified to obtain the possible optimal solution to the problem. In conclusion, the computation procedure used could handle the uncertain quadratic programming problem practically. For future research direction, it is recommended to cover the constraints, both for equality and inequality constraints, in solving the quadratic programming problems.

\section{ACKNOWLEDGMENT}

The authors would like to thank the Ministry of Education Malaysia (MOE) for supporting this research under the Fundamental Research Grant Scheme Vot No. FRGS/1/2018/STG06/UTHM/02/5 and partially sponsored by Universiti Tun Hussein Onn Malaysia.

\section{References Références Referencias}

1. Carl, B.A., Moskowitz, H. and Furtan, H., Quadratic Programming Applications, Omega, 1977, Vol. 5, Iss. 1, pp. 43-55.

2. Guo, P. Huang, G.H. and Li, Y.P., Interval Stochastic quadratic Programming approach for Municipal Solid Waste Management, Journal of Environment Engineering and Science, 2008, Vol. 7, Iss. 6 pp. 569-579.

3. Sunarsih, S., Multi-Period Quadratic Programming Model for Sewon-Bantul Facultative Ponds Optimization, Advances in Science, Technology and Engineering System Journal, 2019, Vol. 4, Iss. 6, pp. 397-401.

4. Zhou, J., A New Spatial Branch and Bound Algorithm for Quadratic Program with One Quadratic Constraint and Linear Constraints, 
Mathematical Problems in Engineering, Vol. 2020, Article ID 5717301, 2020, 8 pages.

5. Shi, Y., Peng Y., Kou G. and Chen, Z., Classsifying Credit Card Accounts for Business Intelligence and Decision Making: A Multiple-Criteria Quadratic Programming Approach, International Journalof Information Technology and Decision Making, 2005, Vol. 4, pp. 581-599.

6. Cui,X. Zhu, S., Li, D. and Sun, J., Mean-Variance Portfolio Optimization with Parameter Sensitivity Control, Optimization Methods and Software, 2016, Vol. 31, Iss.4, pp. 755-774.

7. Zhao, W., Wang, L., Yin, Y. Wang, B. and Tang, Y., Sequential Quadratic Programming Enhanced Backtracking Search Algorithm, Frontiers of Computer Science, 2018, Vol. 12, pp. 316-330.

8. Gabr, W.I., Quadratic and Nonlinear Programming Problems Solving and Analysis in Fully Fuzzy Environment, Alexandria Engineering Journal, 2015, Vol. 54, Iss. 3, pp. 457-472.

9. Mirmohseni, S.M. and Nasseri, H., A Quadratic Programming with Triangular Fuzzy Numbers, Journal of Applied Mathematics and Physics, 2017, Vol. 5, 2218-2227.

10. Taghi-Nezhad, N.A. and Taleshian, F., A Solution Approach for Solving Fully Fuzzy Quadratic Programming Problems, Journal of Applied Research on Industrial Engineering, 2018, Vol. 5, No. 1, pp. 50-61.

11. Barik, S.K. and Biswal, M.P., Probabilistic Quaratic Programming Problems with Some Fuzzy Parameters, Advances in Operations Research, Vol. 2012, Article ID 635282, 2012, 13 pages.

12. Mittal, A., Gokalp, C. and Hanasusanto, G.A., Robust Quadratic Programming with Mixed-Integer Uncertainty, INFORMS Journal of Computing, 2019, Vol. 32, No. 2, pp. 201-218.

13. Chong, E.K.P. and Zak, H.S., An Introduction to Optimization, $4^{\text {th }}$ Ed., John-Wiley \& Sons, Inc., 2013.

14. Luenberger, D.G. and Ye, Y., Linear and Nonlinear Programming, $4^{\text {th }}$ Ed., New York: Springer, 2016.

15. Sinha, S.M., Mathematical Programming: Theory and Methods, Elsevier Science, 2006.

16. Nocedal, J. and Wright, S.J., Numerial Optimization, New York: Springer, 1999.

17. Anderson, D.R., Sweeney, D.J., Williams, T.A. Camm, J.D. and Cochran, J.J., An Introduction to Management Science: Quantitative Approaches to Decision Making, 14 $4^{\text {th }}$ Ed., Cengage Learning, 2015.

18. Johnathan, M., Risk Simulator User Manual, Real Option Valuation Press, 2015.

19. Johnathan, M., Modeling Risk: Applying Monte Carlo Risk Simulation, Strategic Real Optiions, Stochastic Forecasting, Portfolio Optimization, Data Analytics, Business Intelligence, and Decision Modeling, Real Option Valuation Press, 2015.
20. Johnathan, M., Advanced Analytical Models: Applications in ROV Modeling Toolkit, $2^{\text {nd }}$ Ed., Create Space Independent Publishing Platform, 2016. 